\title{
NEUES MATERIAL ZUR GESCHICHTE DER GENFER ESPÉRANCE ${ }^{1}$
}

Unlängst mir zugänglich gemachtes und von mir neuaufgefundenes Archivmaterial wirft zusätzliches Licht auf die Geschichte des Genfer Tageblattes „L'Espérance”.

In einem früheren Aufsatz in dieser Zeitschrift ${ }^{2}$ teilte ich mit, dass Armand Lévy, Chefredakteur der „Espérance”, nachdem die Zeitung im Februar I 86I eingegangen war, sie in Frankreich wieder zu veröffentlichen suchte. Er wandte sich an den Innenminister Persigny um die Genehmigung, die Zeitung in Paris drucken zu lassen. „Im Monat Mai I 86r”, schrieb ich, „erhielt Lévy die gewünschte Genehmigung. Die 'Espérance' ist aber trotzdem, aus mir unbekannten Gründen, nicht wieder erschienen." 3 Im Lichte des neuerschlossenen Materials soll diese Mitteilung ergänzt und berichtigt werden.

In dem Dossier $F^{18} 545$ der Archives Nationales zu Paris ${ }^{4}$ findet sich in der Tat ein Arrêté ${ }^{5}$, wodurch „, $\mathrm{M}^{\mathbf{r}}$ Armand Lévy est autorisé à publier à Paris le journal international intitulé: l'Espérance". Auf Grund dieses Dokumentes konnte ich annehmen, dass Lévy die Genehmigung erhalten hat. Ein anderes, mir damals unbekanntes Dossier über Lévy ${ }^{6}$ berechtigt aber zur Annahme, dass jenes Arrêté nur ein Entwurf war, den eine Bürostelle für eine höhere Instanz ausgearbeitet hatte, die ihn jedoch nicht angenommen hat. In diesem Dossier ist nämlich ein Brief Lévys vom 30. Juni I86I vorhanden, in dem er darüber klagt, dass die Genehmigung noch immer nicht angekommen sei. ${ }^{7}$ Ferner enthält es eine Note des Oberkammerherrn über Lévys Gesuch, ${ }^{8}$ eine Anfrage des Innenministers beim Polizei-

1 Die vorliegende Arbeit wurde am I7. Oktober ${ }_{1963}$ abgeschlossen.

2 E. Silberner, Zur Verfasserschaft der Berichte "aus Deutschland” in der Genfer Espérance, in: International Review of Social History, 7. Bd., 1962, S. 441-445.

3 Ebenda, S. 443.

${ }^{4} F^{18}$ 549: Presse étrangère. Dossiers des journaux introduits en France, classés par ordre alphabétique. Espérance.

5 Datiert: Mai I86ז, ohne Tagesdatum.

6 Archives Nationales. Paris. F $^{18}{ }_{28} \mathrm{I}$ : Bureau de presse. Dossier personnel d'Armand Lévy. Auf dieses Dossier machte mich Herr Marc Vuilleumier, Genf, aufmerksam, dem ich hier meinen aufrichtigsten Dank ausspreche.

7 Armand Lévy an Napoleon III., 30. Juni I 86I (vgl. Anhang, Nr. 3). Dieser Brief wurde an den Innenminister weitergeleitet (vgl. Anhang, Nr. s).

8 Vgl. Anhang, Nr. I. 
präfekten über Lévy, ${ }^{1}$ eine nicht besonders günstige Antwort des Polizeipräfekten $^{2}$ und ein Memorandum des Innenministeriums ${ }^{3}$ aus dem geschlossen werden darf, dass die Genehmigung verweigert wurde, weshalb auch die „Espérance”, trotz aller Anstrengungen Lévys, in Frankreich nicht erscheinen konnte.

Das neuerschlossene Material enthält auch eine Erklärung Hessens über seine Mitarbeit an der "Espérance”.4 Darin heisst es, er habe von Oktober bis Ende November 1859 jeden zweiten Tag eine Korrespondenz „aus Deutschland” eingesandt, aber von Dezember I 859 bis Mai I860 inklusive dem Blatte täglich Berichte geliefert, und zwar abwechselnd eine "deutsche" und eine Pariser Korrespondenz. ${ }^{5}$

Wird Hess' Erklärung wörtlich genommen, so hat er die folgende Zahl von Korrespondenzen eingesandt: Im Oktober 1859 - I (es erschien in diesem Monat nur eine Nummer des Blattes), November I 859 (30 Tage) - is, Dezember i 859 bis April i 860 (I 2 Tage) - I 5 , Mai i 860 - etwa 20, also zusammen etwa i 88 Artikel.

Davon sind 89 Korrespondenzen „aus Deutschland”. 6 Aus Hess' Erklärung geht hervor, dass er auch solche „aus Deutschland” einsenden konnte, die nur aus Zeitungsausschnitten bestanden. Die „Espérance" brachte bis Ende Mai I 860 sechzehn derartige Beiträge;" sie dürfen Hess zugeschrieben werden, da er doch der einzige „deutsche" Korrespondent des Blattes war. Alles in allem sandte er also der „Espérance” ros Berichte „aus Deutschland”.

Zieht man von der Gesamtzahl der I 88 Artikel, die Hess eingesandt zu haben scheint, seine ros Korrespondenzen ,aus Deutschland” ab, so bleiben 83 übrig, die er als Pariser Berichte dem Blatte zukommen

1 Vgl. Anhang, Nr. 2.

2 Vgl. Anhang, Nr. 4.

3 Vgl. Anhang, Nr. 6.

4 Vgl. Anhang, Nr. 7.

5 Die letzten zwei Korrespondenzen von Hess („Berlin, 3 juin” und „Berlin 6 juin” in den Nrn. I 58 und I $_{3}$ der „Espérance” vom 8. resp. I3. Juni 1860) sind wohl von der Redaktion des Blattes nachdatiert.

6 Und zwar: 84 Korrespondenzen, die ich in der International Review of Social History, I 962 , S. 444, aufzähle; 4 andere, die ich ebendort (S. 444, Anm. I) als problematisch bezeichne, die abex auf Grund der Hess'schen Erklärung als die seinigen betrachtet werden können; und die einzige von Hess unterzeichnete Korrespondenz: „La Fête séculaire de Frédéric Schiller" ("L'Espérance" vom 3. November 18 59 ).

${ }^{7}$ "L'Espérance", 1859: Nr. 9, I1, 17, (zwei Korrespondenzen in derselben Nummer), 2I, 22, 24, 33, 47, 50 ("Autre correspondance”, S. 2, Kol. 5): 53 ; I 860: Nr. 6, I I, I6, $45,66$. Nicht inbegriffen sind 4 Korrespondenzen ,aus Deutschland" („L'Espérance”, I 860 : Nr. 27, 47 [S. 3, Kol. 5], 50, 75), die ausschliesslich Auszüge aus den Zeitungen: „Dziennik poznanski" und „Czas” enthalten, da Hess die polnische Sprache nicht beherrschte. 
liess. Es ist aber eine Sache, wieviel Berichte er geschickt, und eine ganz andere, wieviel davon die Redaktion der Zeitung gedruckt hat. Bekanntlich war der Redakteur des Blattes, Wladyslaw Mickiewicz, gerade mit Hess' Pariser Korrespondenzen sehr unzufrieden, ${ }^{\mathbf{1}}$ so dass es nicht zu verwundern wäre, wenn er einen gar nicht geringen Teil davon beseitigt hätte, obwohl das Honorar auch für nicht angenommene Berichte bezahlt werden musste. Wohlgemerkt ist in der etwas sonderbaren Erklärung Hessens nur von eingesandten, nicht aber von erschienenen Korrespondenzen die Rede! Mit anderen Worten: das vorhandene Quellenmaterial erlaubt nicht, die genaue Zahl der Pariser Korrespondenzen von Hess festzustellen.

So bleibt nichts anderes übrig als die Berichte aller Pariser Korrespondenten in der „Espérance” gründlich zu lesen und diejenigen auszusondern, die ihrem Inhalte und Stile nach Hess zugeschrieben werden können. Dies habe ich getan, wenn auch mit geringem Erfolg: nur sechs Pariser Korrespondenzen scheinen mir sicher aus Hess' Feder zu sein $;^{2}$ dreizehn würde ich als höchstwahrscheinlich von ihm bezeichnen, ${ }^{3}$ während ich von fünfundvierzig (die es überflüssig wäre hier aufzuzählen) sagen würde, dass sie wahrscheinlich von ihm stammen. ${ }^{4}$

Man wird nun fragen, warum Inhalt und Stil nicht genügen, um mehr Pariser Korrespondenzen von Hess herauszufinden als es mir gelungen ist. Die Antwort ist nicht schwer. Der Inhalt bietet keinen genügenden Masstab, weil auch die anderen Korrespondenten der „Espérance" sich über aktuelle politische Probleme ähnlich wie Hess äusserten. Was nun den Stil anbelangt, so ist zu bemerken, dass Hess' nicht ganz adäquates Französisch offenbar einer redaktionellen Revision unterzogen wurde, wodurch wahrscheinlich seine Pariser Korrespondenzen nicht wenig an Prägnanz verloren. Man hat den Eindruck, dass die Redaktion der „Espérance” diese Korrespondenzen viel gründlicher feilte als seine Berichte ,aus Deutschland”, weil die

1 Vgl. W. Mickiewicz an A. Lévy, I I. Februar I860, in International Review of Social History, I962, S. 444 .

2 Sie erschienen in "L'Espérance" 1860: Nr. 4 (4. Januar, S. I, Kol. 4-5), Nr. I 2 ( I 2. Januar, S. I, Kol. 5-S. 2, Kol. I), Nr. 24 (24. Januar, S. 2, Kol. 2-3), Nr. 44 (13. Februar, S. 2. Kol. I-2), Nr. 94 (3. April, S. I, Kol. 3-5) und Nr. I 5 (25. April. S. 2, Kol. I-2).

3 „L'Espérance”, I 859: Nr. 31, S. 2, Kol. I ("Autre correspondance”); Nr. 49, S. I; Nr. 50, S. 1; Nr. 53, S. I; Nr. 55, S. I; Nr. 57, S. I; Nr. 61, S. I („Correspondance particulière", Paris, 28. Dezember); 1860: Nr. 10, S. 2; Nr. 16, S. 2; Nr. 27, S. 2; Nr. 35, S. 2 ; Nr. 46, S. 2 ; Nr. 98 , S. I.

4 Nur in einem Falle gelang es mir, allerdings in der Gruppe der Hess'schen Korrespondenzen ,aus Deutschland”, einen Bericht auf Grund seines Rohentwurfes festzustellen: die Korrespondenz, die in Nr. I I der „Espérance” vom I. Juni 1860 erschienen ist, enthält mindestens zwei Sätze aus dem Hess'schen Ms. B I 29 (unveröff., Internationales Institut für Sozialgeschichte, Amsterdam). 
ersteren offensichtlich ihr Missbehagen erweckten, während sie an den letzteren nichts auszusetzen hatte.

Die hier mehrmals angeführte Hess'sche Erklärung' zeigt, dass ihm seine Mitarbeit an der „Espérance” keinen grossen Gewinn eingebracht hat. Bekanntlich hat er zur gleichen Zeit auch an der Augsburger „Allgemeinen Zeitung” mitgearbeitet. ${ }^{2}$ Obwohl sie viel zeitraubender war als die Mitarbeit an der „Augsburgerin”, wurde die Berichterstattung für das Genfer Blatt geringer bezahlt. Als Hess, wie oben erwähnt, jeden zweiten Tag der „Espérance” eine Korrespondenz lieferte, wurde er dafür mit $\mathrm{I} 20 \mathrm{frs}$. im Monat honoriert. $\mathrm{Ab}$ Dezember 1859 wurde ihm für tägliche Berichte ein Monatshonorar von 200 frs. angeboten, das ihm aber nur bis zum Februar regelmässig ausgezahlt wurde. Als er seine Mitarbeit an der „Espérance" beendet hatte, blieb ihm die Zeitung 500 frs. schuldig.

\section{$A N H A N G$}

NOTE DU GRAND CHAMBELLAN DE S.M. 3 I MAI I 86 I. $^{3}$

M. Armand Lévy soumet à l'Empereur le projet de fondation d'un Journal napoléonien et populaire à Paris qui ferait ressortir tout ce que le Gouvernement de l'Empereur a déjà fait dans l'intérêt des masses, et pressentirait l'opinion sur les améliorations les plus pratiques et les plus immédiatement réalisables.

Plus les anciens partis redoublent leurs attaques contre le Gouvernement de l'Empereur, plus les masses populaires placent en lui leur espoir. Ce journal soutiendrait ces bonnes dispositions et ferait sentir

\footnotetext{
1 Vgl. Anhang, Nr. 7.

2 Vgl. Silberner, The Works of Moses Hess, an Inventory of his Signed and Anonymous Publications, Manuscripts and Correspondence, Leiden I958, S. 22-33. Ich benütze diese Gelegenheit um hier bisher unbekannte Briefe oder Briefauszüge zu veröffentlichen (vgl. Anhang, Nrn. 9-II), aus denen zu erfahren ist, wie Hess dazu kam, für die Augsburger "Allgemeine Zeitung", an der unter anderen auch Wilhelm Liebknecht mitgearbeitet hat, Berichte zu verfassen. - Herrn Tilmann Krömer vom Schiller-Nationalmuseum danke ich für seine Hilfsbereitschaft, besonders während meines Besuches in Marbach im August ${ }_{1963}$.

3 So betitelt in dem „Bordereau des Pièces" dieses Dossiers (Archives nationales. Paris.

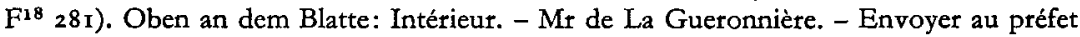
de police pour lui demander son avis. - Très urgent. Links am Rande: 3 I mai.
} 
au Peuple que c'est sur le Gouvernement de l'Empereur qu'il peut le plus sûrement compter.

Un Journal écrit dans cet esprit fait défaut dans la Presse Parisienne. Il exercerait une grande influence sur les classes ouvrières qui seraient admises à faire connaître les améliorations les plus généralement désirées.

Au moment des élections, ce journal pourrait être très utile.

Reçu par ordre de l'Empereur par le Grand Chambellan.

[Ohne Unterschrift.]

DER INNEMINISTER AN DEN POLIZEIPRÄFEKTEN ${ }^{1}$

Paris, le 2 Juin $186 r$.

M. le P[réfet], le $\mathrm{Sr}$ Armand Lévy, prop[riétai]re, demeurant rue de l'Est, No 17 , sollicite l'autorisation de créer, à Paris, un journal politique sous le titre de "l'Espérance".

Avant de statuer sur cette demande, je vous prie, M. le P[réfet], de vouloir bien me donner, d'urgence, les renseignements de nature à m'éclairer sur la moralité et les antécédents politiques du Sr Armand Lévy, ses garanties pécuniaires et les chances de succès qu'il peut avoir. Agréez etc....

[Ohne Unterschrift.]

\section{III}

LETTRE À L'EMPEREUR DE M. A. LÉVY, TRANSMISE PAR LE CABINET DE S. M. ${ }^{2}$

[Stempel:] Cabinet de l'Empereur

Arrivée le 2 Juillet 186 I

Sire,

M. le duc de Bassano ${ }^{3}$ a eu la bonté de mettre sous les yeux de Votre Majesté le projet de publication à Paris du journal l'Espérance.

${ }^{1}$ Archives nationales. Paris. $\mathrm{F}^{18}$ 28I. Links am Rande des Blattes: minute. Dass dieser Brief vom Innenminister ist, folgt aus der Antwort des Polizeipräfekten vom I. Juli I 86I, vgl. Anhang, Nr. 4.

2 So betitelt in dem „Borderau des Pièces” dieses Dossiers (Archives nationales. Paris. $\mathrm{F}^{18}$ 28I).

3 Der Oberkammerherr (Grand Chambellan). 
S. Ex. [le duc de Bassano] m'a fait l'honneur de me faire connaître que Votre Majesté avait vu ce projet avec bienveillance.

Mais l'autorisation n'est pas encore venue \& le temps passe.

Il y a pourtant une utilité réelle à ce qu'il y ait le plus vite possible à Paris un organe Napoléonien \& populaire.

C'est assurément l'un des moyens les plus efficaes de soustraire les masses aux influences funestes des anciens partis, de calmer certaines impatiences \& d'assurer un progrès sagement libéral \& vraiment patriotique.

Mes amis \& moi nous avons pensé que le grand devoir national nous commandait de faire dès à présent ce que Carnot $\&$ les républicains comme lui n'ont fait malheureusement qu'à la dernière heure. ${ }^{1}$

Votre Majesté en sera plus forte \& la France plus assurée de son lendemain.

Daigne Votre Majesté, agréant le projet dont il s'agit, donner l'ordre d'en hâter l'exécution \& croire au profond respect de celui qui a l'honneur d'être,

De Votre Majesté,

Sire,

Le très humble, très obéissant

$\&$ très dévoué serviteur

Paris, I7 Rue de l'Est

30 juin $\mathrm{I} 86 \mathrm{I}$

Armand Lévy

IV

BERICHT DES POLIZEIPRÄFEKTEN ÜBER ARMAND LÉVY2

Cabinet

$\mathrm{du}$

Préfet de Police.

Paris, le $\mathrm{I}^{\text {er }}$ Juillet $\mathrm{r} 86 \mathrm{I}$

I er Bureau.

Monsieur le Ministre, ${ }^{3}$

J'ai l'honneur de vous adresser les renseignements que vous m'avez demandés, par lettre du 2 Juin courant, sur le Sr Lévy, Armand,

1 In dem Briefe Lévys an den Innenminister Persigny vom 27. März I 86 I (ebenda, F ${ }^{18}$ 549) heisst es: „L'Espérance a sans cesse combattu toute pensée de restauration \& de coalition. Sa maxime a été à cet égard: Si les Démocrates comme Carnot eûssent fait en I 809 ce qu'ils ont fait en I 8 I 4, la France n'eût subi l'invasion."

2 Archives nationales. Paris. $F^{18} 28 \mathrm{r}$.

${ }^{3}$ Unten am Blatte: A S. Exc. Mr le Ministre de l'Intérieur. 
propriétaire, qui sollicite l'autorisation de fonder un journal politique sous le titre de „l'Espérance”.

Le Sr Lévy (François-Armand-Théodore), est né le I 2 Mars I 827 , à Précy-sous-Thil (Côte-d'Or). Il demeure à Paris, depuis 14 ans, rue de l'Est No i 7 .

Il s'est de tout temps fait remarquer par l'exaltation de ses principes politiques et par ses relations avec les chefs de la démocratie.

Il était étudiant en droit lorsque la révolution de i 848 éclata. Il fonda un club dit „Club de la Redoute”,", dont Pierre Leroux, Madier de Montjau, ${ }^{2}$ Bernard, ${ }^{3}$ etc., suivaient les séances, où il prononça plusieurs discours très-violents, à la suite desquels il fut condamné une première fois à $100 \mathrm{fr}$. et une seconde fois à $200 \mathrm{fr}$. d'amende.

Il fut arrêté le 3 Juin de la même année sous l'inculpation d'avoir participé à l'insurrection du is Mai, et une ordonnance de non lieu a été rendue en sa faveur, le is Juillet suivant. Poursuivi de nouveau à la suite des événements du ${ }_{3} 3$ Juin ${ }_{1} 849$, il se réfugia en Belgique où il continua ses relations avec les principaux chefs de la démagogie, puis il revint en France, où il se fit remarquer dans les clubs par son ardeur révolutionnaire; il était en 1850 délégué au comité électoral socialiste du $\mathrm{I}^{\mathrm{e}}$ arrondissement. A la fin de l'année suivante, on signalait les discours qu'il avait prononcés dans plusieurs réunions électorales, comme empreints des sentiments les plus exaltés. Il fut poursuivi, la même année, sous l'inculpation d'avoir répandu dans son pays natal des ouvrages socialistes. Un mandat d'amener fut même décerné contre lui à cette occasion, mais le Sr Lévy parvint à se soustraire à toutes les recherches.

Depuis cette époque jusqu'à ce jour, il ne paraît pas s'être livré à une propagande révolutionnaire active comme par le passé. Mais il s'est posé dans l'arrondissement de Semur comme chef de la démocratie, et dans toutes les élections qui ont eu lieu il a été le candidat de l'opposition.

Il a fondé, en 1859, à Genève, un journal pratique [sic] intitulé „l'Espérance”, dont je crois devoir joindre un exemplaire 4 à ma lettre pour vous mettre en mesure d'apprécier la ligne de conduite politique qu'il s'est tracée dans ce journal et qu'il a probablement l'intention de suivre dans la nouvelle feuille qu'il sollicite l'autorisation de fonder.

1 In seinem Artikel über den „Club de la Redoute” erwähnt Alphonse Lucas(Les Clubs et les Clubistes, Paris I85 I, S. 197) Lévy nicht.

2 Wohl Noël-François-Alfred Madier de Monjau (1814-1892). Rechtsanwalt und französischer Politiker.

3 Aristide-Martin Bernard (1 808-1 883), französischer Politiker.

4 Liegt nicht vor. 
Sa conduite et sa moralité n'ont donné lieu à aucune observation défavorable, et il ne figure sur les registres des sommiers judiciaires qu'à raison des poursuites pour complot énoncées plus haut.

Il est dans une position de fortune aisée. Il possède des propriétés dans le département de la Côte-d'Or, et ses amis politiques de divers pays étrangers lui fourniraient, dit-on, une partie des fonds nécessaires. Veuillez, Monsieur le Ministre, agréer l'hommage de mon respect.

Le Préfet de Police

[Unterschrift unlesbar.]

\section{V}

CABINET DE L'EMPEREUR AN DEN INNENMINISTER ${ }^{1}$

Cabinet

de

L'Empereur

Palais des Tuileries, le 8 Juillet I861.

Monsieur le Ministre,

J'ai l'honneur de vous transmettre sous ce pli, d'après les ordres de l'Empereur, une demande de M. Armand Lévy² qui sollicite l'autorisation de publier un journal.

Agréez, Monsieur le Ministre, l'assurance de ma haute considération. Le S[ou]s Chef du Cabinet de l'Empereur

M. le Ministre de l'Intérieur

[Unterschrift unlesbar.]

\section{VI}

MEMORANDUM DES INNENMINISTERIUMS ${ }^{3}$

Ministère

de l'Intérieur

Paris, le [blanko] i 86[blanko]

\section{Cabinet}

du Conseiller d'Etat

en mission

Translation à Paris du

journal suisse L'Espérance

Mr Armand Lévy, demandeur.

Démocrate ardent, quoiqu'il professe personnellement un grand respect pour la dynastie napoléonienne.

${ }^{1}$ Archives nationales. Paris. $\mathrm{F}^{18} 28 \mathrm{I}$.

${ }^{3}$ Archives nationales. Paris. $F^{\mathbf{1 8}} \mathbf{2 8 1}$.

2 Vom 30 Juni 1861, vgl. Anhang, Nr. 3. 
Renseignements peu favorables de la préfecture de police, au point de vue politique. ${ }^{1}$

Avis contraire du chef de $\mathrm{B}[\mathrm{ure}] \mathrm{au}$

[Ohne Unterschrift.]

[Links am Rande mit rotem Bleistift:] Audience 22 [juillet.]

[Unten am Blatte in derselben Handschrift wie die obige Randbemerkung, mit rotem Bleistift:]

prendre les ordres de S. E[x].

Refus notifié le 22 juillet $\mathrm{r} 86 \mathrm{r}$.

\section{VII}

HESS' ERKLÄRUNG ÜBER SEINE MITARBEIT

AN DER, ,ESPÉRANCE"2

L'année passée, avant de commencer le journal „L'Espérance” qui se publie à Genève depuis Octobre 1859, M. Armand Lévy est venu chez moi et m'a offert 120 francs par mois pour lui faire tous les deux jours des extraits des journaux allemands en forme de correspondance, ou autre. J'ai accepté l'offre et commencé mes travaux que j'ai continué[s] de la sorte jusqu'à la fin du mois de Novembre. Depuis cette époque M. Lévy m'a offert 200 francs par mois pour une correspondance quotidienne, en faisant un jour une correspondance de Paris et le lendemain une correspondance d'Allemagne. J'ai fait ces correspondances jusqu'à la fin du mois de Mai. On m’a payé mon honoraire jusqu'à la fin du mois de Février regulièrement. Depuis lors je n'ai reçu que so francs de M. Armand Lévy au commencement du mois d'Avril. Vers la fin de ce mois $j$ 'ai fait une traite de 350 francs sur Genève qui me fut renvoyée avec protêt. Les frais de protêt s'élevaient à i 2 francs 90 cts. Je ne compte que i37.10 fs., au lieu de fs. 200, pour le mois de Mai, parce que, dans ce mois, j'ai manqué quelques jours d'envoyer des correspondances. On me doit donc aujourd'hui 500 Francs. M. Armand Lévy, auquel j'ai présenté la note ci-jointe, ${ }^{3}$ l'a reconnue, en me priant seulement de ne pas faire mention des frais du protêt, et de reporter les fs. I $2.9^{\circ}$ sur mon honoraire du mois de Mai. M. Armand Lévy ne niera pas ces faits.

M. Hess

Paris, le I 2 Juillet 1860 33, rue de l'Est

${ }^{1}$ Gemeint ist der Bericht des Polizeipräfekten vom I. Juli $x$ 86r, vgl. Anhang, Nr. 4.

${ }^{2}$ Original im Institut für Marxismus-Leninismus, Moskau; oben am Blatt: Rj[azanov?] 1076; Adressat unbekannt. Mikrofilm im Istituto Giangiacomo Feltrinelli (Mailand), dem hier für die Mitteilung des Dokumentes Dank gesagt wird.

${ }^{3}$ Liegt nicht vor. 


\section{VIII}

HERMANN ORGES AN

BARON JOHANN GEORG COT'TA VON COTTENDORFl

(Auszugsweise)

Augsburg, den 7. Februar I 859.

„Herr Hunziker ${ }^{2}$ hat Paris verlassen, wir bedürfen aber dort wohl noch eines Correspondenten. Man empfiehlt mir sehr einen Herrn Hess, der unabhängig von seinem Vermögen lebt, Nationalökonom und gründlich wissenschaftlich gebildet ist. Er ist nichts weniger als Tagesschriftsteller, aber Herr Hunziker ist voll seines Lobes, besonders seiner deutschen Gesinnung. Herr Hess hätte wie Hunziker nur gelegentlich wissenschaftliche etc. Erscheinungen zu besprechen."

HERMANN ORGES AN

BARON JOHANN GEORG COTTA VON COTTENDORF

(Auszugsweise)

Augsburg, den 16. Februar I859. „Soll ich Herrn Hess um Fortsetzung seiner Einsendungen bitten?4 Er hat eine Analyse des Lebens der Herzogin von Orléans ${ }^{5}$ geschickt."

\section{$\mathrm{X}$}

HERMANN ORGES AN MOSES HESS 6

[Datum des Poststempels:]

Augsburg, 17. Feb[ruar][18] 9 .

Hochgeehrtester Herr!

Unsern herzlichsten Dank für Ihre Zusendungen; von Baron Cotta noch keine Antwort; ich erwarte sie täglich.

1 Original im Schiller-Nationalmuseum (Cotta-Archiv) in Marbach. Hermann Orges (1821-1874), Publizist, verantworlicher Redakteur der Augsburger „Allgemeinen Zeitung", deren Inhaber Baron Johann Georg Cotta von Cottendorf (1796-1 863) war.

2 Uber J. Hunziker liegen keine biographischen Daten vor.

3 Original im Schiller-Nationalmuscum. (Cotta-Archiv).

4 Im Original Punkt statt Fragezeichen.

${ }^{5}$ Gemeint ist eine Besprechung des anonymen Buches: Madame la duchesse d'Orléans, Hélène de Mecklembourg-Schwerin. [Par la marquise Georges d'Harcourt.] Paris, MichelLévy frères, 1859. In-80. $239 \mathrm{~S}$. Sechs Auflagen des Buches erschienen im Jahre 1859. 6 Original im Institut für Marxismus-Leninismus, Moskau; Rj I077. Mikrofilm im Istituto Giangiacomo Feltrinelli, dem ich für die Mitteilung dieses Briefes zu Dank verpflichtet bin. 
Analyse des Lebens d[er] H[erzogin]v.O[rléans] wird gegeben werden. ${ }^{1}$ Halten Sie ja Ihre Verbindung [mi]t uns so geheim als möglich. Lassen Sie sich von Herrn Hefner $^{2}$ von unseren Tendenzen unterrichten. ${ }^{3}$ Ihre nächste Musse bitte ich zu einer Reihe von Artikeln zu benutzen, die an einen Artikel von mir, der dieser Tage erscheint, anknüpfen: Zur innern Politik des 2 t. Dezembers. Ihre Aufgabe wird es sein, nachzuweisen, dass Frankreich in der Auflösung u[nd] einem chronischen Siechtum begriffen ist. - Sie müssen da vom Nationalcharakter ausgehen, daran erinnern, dass miscbblütige Völker, die auf den Trümmern einer alten Cultur (der römischen) fortkauend sich rascher entwickeln, aber auch früher dafür abtreten. Sie müssen durchführen, wie namentlich der $2^{\text {t. }}$ Dezember den letzten Lebenskern verbraucht hat. Schilderung des zerfahrenen, verwahrlosten, verkommenen Familienlebens, die Herrschaft der Lorette, die ungeniertesten[?] ${ }^{4}$ Erziehungsprincipien, das jeune France des Quartier latin, die Bestechlichkeit der Beamten, die Liederlichkeit der Litteratur, der Mangel an wirklichen Zwecken, Männern, die effektive Armut an Charakteren, die Verbrechen. [Hess' Anmerkung:] Merde!

Schreiben Sie meinetwegen eine Skizze des Lebens des Mädchens, des Mannes. Skizziren Sie den Studenten, den Arbeiter, die Börsenwelt, etc., was Sie wollen, nur müssen Sie nie den Augenpunkt verlieren, stets sich daran erinnern, dass es darauf ankommt in Deutschland die Überzeugung zu schaffen, dass Frankreich ein sich auflösendes Land ist, auf dessen Convulsionen wir uns gefasst machen müssen.

Mit grösster Hochacht[ung] Euer Hochwohlg[eboren] ganz erge[bener] H.O. [Hess' Anmerkung:] Lump!

\section{[Folgende Seite:]}

Verte!

\footnotetext{
${ }_{1}^{1}$ Orges hat die Hess'sche Besprechung des Lebens der Herzogin von Orléans (vgl. Anhang, Nr. 9) nicht veröffentlicht. Die anonyme Rezension dieses Werkes in der Augsburger „Allgemeinen Zeitung” (Beilage zu Nr. 54 vom 23. Februar i 859, S. 866 f.) stammt, wie aus dem Redaktionsexemplar im Schiller-Nationalmuseum (Cotta-Archiv) zu exsehen ist, von Wilhelm von Voigts-Rhetz.

${ }^{2}$ Leopold Häfner (geb. I 820), langjähriger Pariser Korrespondent der Augsburger "Allgemeinen Zeitung", Emigrant aus der Wiener Oktoberrevolution 1848, stand seit I85 I im Dienste der österreichischen und französischen Polizei, wurde aber schon im folgenden Jahre entlarvt (vgl. darüber: „Karl Vogt und die Allgemeine Zeitung” [anonym], in National-Zeitung vom 22. Januar 1860, Morgenausgabe). Quellenangaben über seine Polizeitätigkeit, bei Boris Nikolajewsky, in International Review for Social History, 1. Jg., 1936, S. I68, Anm. Häfner schrieb für sein Blatt stark antinapoleonisch gefärbte Berichte, die „eine lebhaft ausgeprägte deutsche Gesinnung” zeigen (Ed. Heyck, Die Allgemeine Zeitung 1 798-1898, München I 898, S. 197).

3 Orges war ein enragierter Vertreter der österreichischen Vorherrschaft. Vgl. über ihn den soeben angeführten anonymen Aufsatz in der National-Zeitung.

4 Auf meiner Fotokopie nicht sicher lesbar.
} 
Bitte sagen Sie, verehrtester Herr, Herrn Littré1, dass ich die Verstümmelung meines Aufsatzes sehr bedauere. Er hat, wie Sie aus der Einleitung sehen, einen abgestutzten Kopf bekommen. Sie werden gewiss fühlen, dass der erste Satz, der im V[orwort] enthalten, nicht von mir [ist].

In dieser Manier ist viel gestrichen und nur das Skelett geblieben. Ich bin ein besserer Materialist als die Analyse der Comte'schen Werke zu erkennen giebt.

[Hess' Bermerkung:] Dieser B[rie]f, den ich nicht beantworte, soll nur als Dokument der Dummheit und Niedertracht der ruchlosen[?] ${ }^{2}$ Teutomanen dienen.

Monsieur Maurice Hess

33, Rue de l'Est

Paris

\section{XI}

HERMANN ORGES AN

BARON JOHANN GEORG COTTA VON COTTENDORF ${ }^{3}$

(Auszugsweise)

Augsburg, den 22. Februar [1 859] „Herr Alexander [sic] Hess ist ein von einem kleinen Vermögen ganz unabhängig als Privatgelehrter in Paris lebender Deutscber, derselbe wohnt 33 Rue de l'Est. Ich kenne ihn weiter nicht; Herr Hunziker, der Paris für immer verlassen hat und jetzt in Aarau ist, hat ihn empfohlen. Herr Hess ist mit Littré genau bekannt, was ihn jedenfall ehrt. Ausser Herrn Häfner bedürfen wir doch wohl noch eines Korrespondenten für wissenschaftliche Dinge; als solchen erlaube ich mir versuchsweise Herrn Hess zu proponiren."

1 Emile Littré ( $1801-188 x$ ), französischer Sprachforscher und Philosoph.

2 Auf meiner Fotokopie nicht sicher lesbar.

${ }^{3}$ Original im Schiller-Nationalmuseum (Cotta-Archiv) in Marbach. 